Studies on genetic and epigenetic mechanisms of carcinogenesis have led to the discovery of crucial genetic events for many of particular malignancies. This was followed by invention of new therapeutic approaches based on molecular mechanisms underlying cancer development and progression that bears the name of personalised medicine. In the case of gliomas, ascertainment of genetic/ epigenetic markers was the basis for re-classification of tumours that until now depended on histopathological analysis. This article reviews recent advances in personalised medicine and the new World Health Organisation classification of gliomas.

Key words: gliomas, epigenetic mechanisms of carcinogenesis, personalized medicine.

Contemp Oncol (Pozn) 2018; 22 (1A): 1-2 DOI: https://doi.org/10.5114/wo.2018.73872

\section{Personalized medicine in oncology. New perspectives in management of gliomas}

\author{
Justyna Gil*, Izabela Laczmanska*, Karolina A. Pesz, Maria M. Sasiadek \\ Genetics Department, Wroclaw Medical University, Poland \\ * Equal contribution.
}

Personalized cancer medicine is based on molecular profiling of cancer cells. Defining genetic alterations, aside from establishing a precise molecular diagnosis of the tumour and predicting the course of the disease, allows granting an individual patient tailored therapy in accordance to genomic as well as epigenomic characteristics of the tumour [1]. A better understanding of molecular mechanisms underlying cancer development and progression has enabled the development of new therapeutic schemes aimed precisely at particular protein products of pivotal genes [2]. Nowadays, these therapeutic procedures are becoming standard management for an increasing number of cancers [1]. Alterations such as ERBB2 (HER2) amplification in breast cancer, EGFR mutations in non-small cell lung carcinoma, KRAS and $B R A F$ mutations in colorectal cancer or $B C R-A b l$ fusion in chronic myelogenous leukemia are routinely examined. Targeted cancer therapies, which emerged from the results of these studies permitted an increase of treatment effectiveness in oncological patients [2, 3].

Immense progress in our understanding of cancer genetics was made possible through development of molecular techniques. Availability of large scale molecular approaches like next generation sequencing (NGS) and array techniques for chromosomal instability, gene expression or methylation pattern allowed to identify vast numbers of different genetic alterations in each malignancy. These analyses allowed in turn establishing key genetic features to employ as biomarkers for optimization of cancer patients treatment [4].

The significance of personalized medicine is well illustrated by an innovative approach to classification of gliomas published in 2016 by World Health Organization (WHO) [5]. Previous classification of gliomas (WHO, 2007) was based on histologic criteria such as microscopic analyses of hematoxylin and eosin stained cancer specimens, immunohistochemical evaluation of expression of selected proteins and ultrastructural cell differences [6]. These classical approaches proved in discordance with the molecular mechanisms of tumour development and progression and thus did not open the possibility of targeted clinical management of cancer patients [5, 6].

Current revolutionary diagnostics of brain tumours employs both histologic and molecular testing. Clinical utility as prognostic or predictive markers has been proven for mutations in genes such as: IDH1/IDH2 (isocitrate dehydrogenase 1 or 2 genes), ATRX (alpha-thalassemia/mental retardation syndrome X-linked), TERT (telomerase reverse transcriptase) promoter mutations as well as TP53 mutations and 1p/19q co-deletion. Moreover, methylation of the promoter of MGMT (06-methylguanine-DNA methyltransferase) was found to be an interesting predicting marker of response to alkylating agents which are routinely used as chemotherapy of gliomas as well as a marker of potential response to radiotherapy [5].

Our current knowledge of genetic markers of gliomas results from longterm studies that showed alterations crucial for development and progres- 
sion of gliomas' including wide spectrum of genetic changes such as chromosomal aberrations, gene mutations and epigenetic silencing. DNA copy number variations (CNVs) occurring during cell division that result in genomic deletions/duplications belong to the most important mechanisms of tumour suppressors loss-of-function and proto-oncogenes gain-of-function [2]. Array Comparative Genomic Hybridization (aCGH) studies uncovered that the most frequent CNVs in gliomas are 1p, 9p, 10, 10p, 10q, 13q, $19 q, 22 q$ deletions and $7 q$ gains. These studies allowed also to establish a characteristic profile of chromosomal aberrations in gliomas and thus enabled identifying a progression pattern from low to high grade gliomas [2].

IDH1 and IDH2 gene mutations are widely reported in gliomas. IDH1 plays a role in citric acid pathway while IDH2 variants are reported to influence angiogenesis, apoptosis and glucose metabolism [2]. Mutations in codon 132 and 172 in $I D H 1$ and IDH2, respectively, occur in about $70 \%$ of diffuse astrocytic and oligodendroglial gliomas as well as in secondary glioblastomas [4]. IDH mutations are associated with initiation of carcinogenesis in grade II diffuse astrocytomas and grade III anaplastic astrocytomas which are now classified as either: i) IDH-mutant, ii) IDH-wildtype and iii) not otherwise specified (NOS).

TP53 and ATRX gene mutations are a driving force in diffuse and anaplastic astrocytomas while $1 p / 19 q$ co-deletion [resulting from an unbalanced translocation $\mathrm{t}(1 ; 19)$ (q10;p10)] and TERT promoter mutation in oligodendrogliomas [4, 5]. These two different molecular pathways comprise a criterion for distinguishing between astrocytic versus oligodendroglial tumours.

In contrary to diffuse and anaplastic astrocytomas as well as secondary glioblastomas, IDH-wildtype status is characteristic for $90 \%$ of primary glioblastomas (the most frequent central nervous system tumours) [5]. Thus, mutations in IDH gene family in glioblastomas are currently accepted as an important diagnostic, predictive and prognostic biomarker.

Furthermore, in IDH-wildtype glioblastomas genetic alterations such as copy number gains of chromosome 7, monosomy of chromosome 10, mutations in PTEN, homozygous deletion of CDKN2A/P14 ARF, CDKN2B and TERT promoter mutations are frequently observed. Moreover, mutations in TERT promoter have been shown to correlate with patients' survival expectations depending on other genetic alterations such as IDH mutation status, 1p/19q co-deletion and EGFR amplification [2].

Methylation status of MGMT was found to be a promising predictive marker for high grade gliomas. MGMT plays an important role in DNA base excision repair [7]. Thus, MGMT promoter hypermethylation (leading to gene expression downregulation) results in ineffective reparation of DNA alkyl-adducts and in turn, correlates with an enhanced response to both, chemotherapy employing alkylating agents and to radiotherapy [7].

However, as coexistence of MGMT promoter hypermethylation along with 1p/19p co-deletion and IDH1 mutations is frequent in secondary gliomas, it remains unclear which of those genetic events has a more causative role [2].
Recently, several other genetic alterations have been found in gliomas and seems to be a potential new predictive and prognostic biomarkers. Mutations in BRAF, H3K27M and EGFRVIII and fusion of FGFR-TACC will enable to develop better therapeutic strategies [4].

Recent WHO 2016 classification of gliomas has opened up new possibilities in personalized therapy and allows a better understanding of pathogenesis and mechanisms of drug resistance.

Lately, a high potential of a possibility of non-invasive glioma diagnosis before tailored therapy has been also attributed to liquid biopsy.

The authors declare no conflict of interest.

\section{References}

1. Ene $\mathrm{Cl}$, Holland EC. Personalized Medicine for Gliomas. Surg Neurol Int 2015; 6 (Suppl 1): S89-95.

2. van Thuijl HF, Ylstra B, Würdinger T, van Nieuwenhuizen D, Heimans JJ, Wesseling P, Reijneveld JC. Genetics and pharmacogenomics of diffuse gliomas. Pharmacol Ther 2013; 137: 78-88.

3. Lu DY, LU TR, Xu B, Ding J. Pharmacogenetics of cancer therapy: breakthroughs from beyond? Future Sci OA 2015; 1: FSO80.

4. Masui K, Mischel PS, Reifenberger G. Molecular classification of gliomas. Handb Clin Neurol 2016; 134: 97-120.

5. Louis DN, Perry A, Reifenberger G, von Deimling A, et al. The 2016 World Health Organization Classification of Tumors of the Central Nervous System: a summary. Acta Neuropathol 2016; 131: 803-20.

6. Louis DN, Ohgaki H, Wiestler OD, Cavenee WK, Burger PC, Jouvet A, Scheithauer BW, Kleihues P. The 2007 WHO classification of tumours of the central nervous system. Acta Neuropathol 2007; 114: 547.

7. Wick W, Weller M, van den Bent M, et al. MGMT_testing - the challenges for biomarker-based glioma treatment. Nat Rev Neurol 2014; 10: 372-85.

\section{Address for correspondence}

\section{Maria M. Sasiadek}

Genetics Department

Wroclaw Medical University

Marcinkowskiego 1

50-368 Wroclaw, Poland

tel. +48717841258

fax +48 717840063 ,

e-mail: maria.sasiadek@umed.wroc.pl 